\title{
On the Set-Theoretic Strength of Countable Compactness of the Tychonoff Product $2^{\mathbb{R}}$
}

by

\section{Eleftherios TACHTSIS}

Presented by Czestaw RYLL-NARDZEWSKI

Summary. We work in ZF set theory (i.e., Zermelo-Fraenkel set theory minus the Axiom of Choice AC) and show the following:

1. The Axiom of Choice for well-ordered families of non-empty sets $\left(\mathrm{AC}^{\mathrm{WO}}\right)$ does not imply "the Tychonoff product $2^{\mathbb{R}}$, where 2 is the discrete space $\{0,1\}$, is countably compact" in ZF. This answers in the negative the following question from Keremedis, Felouzis, and Tachtsis [Bull. Polish Acad. Sci. Math. 55 (2007)]: Does the Countable Axiom of Choice for families of non-empty sets of reals imply $2^{\mathbb{R}}$ is countably compact in ZF?

2. Assuming the Countable Axiom of Multiple Choice (CMC), the statements "every infinite subset of $2^{\mathbb{R}}$ has an accumulation point", "every countably infinite subset of $2^{\mathbb{R}}$ has an accumulation point", " $\mathbb{R}^{\mathbb{R}}$ is countably compact", and $\mathrm{UF}(\omega)=$ "there is a free ultrafilter on $\omega$ " are pairwise equivalent.

3. The statements "for every infinite set $X$, every countably infinite subset of $2^{X}$ has an accumulation point", "every countably infinite subset of $2^{\mathbb{R}}$ has an accumulation point", and $\operatorname{UF}(\omega)$ are, in ZF, pairwise equivalent. Hence, in ZF, the statement " $2^{\mathbb{R}}$ is countably compact" implies $\mathrm{UF}(\omega)$.

4. The statement "every infinite subset of $2^{\mathbb{R}}$ has an accumulation point" implies "every countable family of 2 -element subsets of the powerset $\mathcal{P}(\mathbb{R})$ of $\mathbb{R}$ has a choice function".

5. The Countable Axiom of Choice restricted to non-empty finite sets, $\left(\mathrm{CAC}_{\mathrm{fin}}\right)$, is, in $\mathrm{ZF}$, strictly weaker than the statement "for every infinite set $X, 2^{X}$ is countably compact".

2010 Mathematics Subject Classification: Primary 03E25; Secondary 03E35, 28A05, 54B10, 54D30.

Key words and phrases: axiom of choice, weak axioms of choice, compactness, countable compactness, Tychonoff products, Lebesgue measure, independence results. 


\section{Notation and terminology}

Definition 1.1.

1. $\mathrm{AC}^{\mathrm{WO}}$ (Form 40 in [4]): Every well-ordered family of non-empty sets has a choice function.

2. DC (principle of Dependent Choices and Form 43 in [4]): If $R$ is a relation on a non-empty set $X$ such that $\forall x \in X, \exists y \in X, x R y$, then there is a sequence $\left(x_{n}\right)_{n \in \omega}$ of elements of $X$ such that $x_{n} R x_{n+1}$ for all $n \in \omega$. (As usual $\omega$ denotes the set of natural numbers and $\mathbb{N}$ denotes the set of positive integers.)

3. CAC (Countable Axiom of Choice, Form 8 in [4]): Every countable family of non-empty sets has a choice function. ( $\mathrm{AC}^{\mathrm{WO}} \Rightarrow \mathrm{DC} \Rightarrow$ CAC; see [5, Theorems 8.1 and 8.2, pp. 120-121]).

4. CMC (Countable Axiom of Multiple Choice, Form 126 in [4]): Every countable family $\mathcal{A}$ of non-empty sets has a multiple choice function, i.e., a function which assigns to every $x \in \mathcal{A}$ a non-empty finite subset of $x$.

5. $\mathrm{CAC}_{\text {fin }}$ (Form 10 in [4]): CAC restricted to families of non-empty finite sets.

6. $\mathrm{AC}(\mathbb{R})($ Form $[79 \mathrm{~A}]$ in $[4])$ : Every family of non-empty sets of reals has a choice function. Equivalently, $\mathbb{R}$ is well orderable.

7. $\mathrm{CAC}(\mathbb{R})($ Form 94 in $[4): \mathrm{AC}(\mathbb{R})$ restricted to countable families of non-empty sets of reals.

8. $\mathrm{CAC}_{\text {fin }}(\mathcal{P}(\mathbb{R}))$ : Every countable family of non-empty finite subsets of $\mathcal{P}(\mathbb{R})$ has a choice function.

9. $\mathrm{CUC}_{\text {fin }}(\mathcal{P}(\mathbb{R}))$ : Every countable family of non-empty finite subsets of $\mathcal{P}(\mathbb{R})$ has a countable union.

10. Let $X$ be a non-empty set. A non-empty collection $\mathcal{F} \subseteq \mathcal{P}(X) \backslash\{\emptyset\}$ is called a filter on $X$ if it satisfies the following two conditions:

(i) if $F_{1}, F_{2} \in \mathcal{F}$, then $F_{1} \cap F_{2} \in \mathcal{F}$,

(ii) if $F \in \mathcal{F}$ and $F \subseteq G$, then $G \in \mathcal{F}$.

A filter $\mathcal{F}$ on $X$ is called free if $\bigcap \mathcal{F}=\emptyset$. A maximal, with respect to inclusion, filter on $X$ is called an ultrafilter on $X$.

11. Let $(X, T)$ be a topological space and let $\mathcal{F}$ be a filter on $X$. We say that the filter $\mathcal{F}$ converges to a point $x \in X$ if every open neighborhood of $x$ belongs to $\mathcal{F}$.

12. $\operatorname{UF}(\omega)$ (Form 70 in [4]): There is a free ultrafilter on $\omega$.

DEFINITION 1.2.

1. Let $X$ be a non-empty set. A non-empty collection $\mathcal{H} \subseteq \mathcal{P}(X) \backslash\{\emptyset\}$ has the finite intersection property, FIP for abbreviation, if for every $\mathcal{Q} \in[\mathcal{H}]^{<\omega}$ (the set of all finite subsets of $\left.\mathcal{H}\right), \cap \mathcal{Q} \neq \emptyset$. 
2. Let $(X, T)$ be a topological space.

(a) $X$ is called compact if every open cover of $X$ has a finite subcover. Equivalently, $X$ is compact if and only if every family of non-empty closed subsets of $X$ with the FIP has a non-empty intersection.

(b) $X$ is called countably compact if every countable open cover of $X$ has a finite subcover. Equivalently, $X$ is countably compact if and only if every countable family of non-empty closed subsets of $X$ with the FIP has a non-empty intersection.

(c) $X$ is called sequentially accumulation point compact if every sequence in $X$ has an accumulation point.

(d) $X$ is called ultrafilter-compact if every ultrafilter on $X$ converges.

3. Let $X$ be an infinite set.

(a) $2^{X}$ will denote the Tychonoff product of the discrete space $2=$ $\{0,1\}$. The family $\{[p]: p \in \operatorname{Fn}(X, 2)\}$, where $\operatorname{Fn}(X, 2)$ is the set of all finite partial functions from $X$ into 2 and $[p]=\left\{f \in 2^{X}\right.$ : $p \subset f\}$, will denote the standard clopen (= simultaneously closed and open) base for the Tychonoff product $2^{X}$.

(b) $\operatorname{TPC}\left(2^{X}\right)$ : The Tychonoff product $2^{X}$ is countably compact.

2. Introduction and some preliminary results. The research on the set-theoretic strength of the statement $\operatorname{TPC}\left(2^{\mathbb{R}}\right)$ (i.e., $2^{\mathbb{R}}$ is countably compact) was initiated in [7] where it was proved that $\operatorname{TPC}\left(2^{\mathbb{R}}\right)$ is not a theorem of ZF set theory (i.e., Zermelo-Fraenkel set theory minus the Axiom of Choice); see Theorems 6 and 7 in [7. Furthermore, in [7, characterizations of the former statement were supplied in terms of the extendability of countable closed filters in $2^{\mathbb{R}}$ as well as in terms of projections of closed subsets of $2^{\mathbb{R}}$ on countably many copies of 2 ; see Theorems 8 and 14 in [7].

Clearly, $\mathrm{TPC}\left(2^{\mathbb{R}}\right)$ is a theorem of $\mathrm{ZF}+\mathrm{AC}(\mathbb{R})$ since $\mathrm{AC}(\mathbb{R})$ is equivalent to the statement " $\mathbb{R}$ is a well orderable set", and in $\mathrm{ZF}$, the Tychonoff product $2^{\aleph}, \aleph$ a well ordered cardinal number, is compact; see [6]. In [7] the following question was posed: Is $\mathrm{TPC}\left(2^{\mathbb{R}}\right)$ provable in the theory $\mathrm{ZF}+$ $\mathrm{CAC}(\mathbb{R})$ ?

In this paper we answer this question in the negative. We achieve our goal by first establishing that, in ZF, $\mathrm{UF}(\omega)$, "for every infinite set $X$, every countably infinite subset of $2^{X}$ has an accumulation point", and "every countably infinite subset of $2^{\mathbb{R}}$ has an accumulation point" are equivalent (see Theorem 3.5). Since for every infinite set $X, \operatorname{TPC}\left(2^{X}\right)$ implies that every countably infinite subset of $2^{X}$ has an accumulation point, we obtain as a corollary (Corollary 3.1) to Theorem 3.5 that, in ZF, "for every infinite set $X, \operatorname{TPC}\left(2^{X}\right)$ " implies $\operatorname{UF}(\omega)$ and in particular $\operatorname{TPC}\left(2^{\mathbb{R}}\right)$ implies $\operatorname{UF}(\omega)$. 
Subsequently, in Theorem 3.6, we prove that the conjunction of $\mathrm{CAC}(\mathbb{R})$ and "every countably infinite subset of $2^{\mathbb{R}}$ has an accumulation point" implies that there is a non-Lebesgue measurable set of reals. Now, Solovay [14] has constructed a model (model $\mathcal{M} 5(\aleph)$ in [4]) of ZF + DC + "every set of reals is Lebesgue measurable", hence $\operatorname{TPC}\left(2^{\mathbb{R}}\right)$ fails in Solovay's model and DC (hence CAC) does not imply $\mathrm{TPC}\left(2^{\mathbb{R}}\right)$ in ZF set theory (Theorem 3.7). Theorem 3.5 enables us to establish (see Theorem 3.8) that $\operatorname{TPC}\left(2^{\mathbb{R}}\right)$ also fails in Feferman's forcing model (see [1] or model $\mathcal{M} 2$ in [4]) in which $\mathrm{AC}^{\mathrm{WO}}$ holds true (see [15]). Therefore, $\mathrm{AC}^{\mathrm{WO}}$ does not imply $\mathrm{TPC}\left(2^{\mathbb{R}}\right)$ in ZF. (However, in every Fraenkel-Mostowski permutation model, CAC implies that for every infinite set $X$, the Tychonoff product $2^{X}$ is countably compact; see Theorem 2.1(2)).

From Theorem 3.7 we also see that the statement "every countably infinite subset of $2^{\mathbb{R}}$ has an accumulation point", hence "every infinite subset of $2^{\mathbb{R}}$ has an accumulation point", is not provable in ZF, and in Theorem 3.9 we show that, in $\mathrm{ZF}$, the latter statement implies that every countable family of two-elements subsets of $\mathcal{P}(\mathbb{R})$ has a choice function.

Regarding the set-theoretic strength of the statement (stronger than $\left.\operatorname{TPC}\left(2^{\mathbb{R}}\right)\right)$ "for every infinite set $X, 2^{X}$ is countably compact", we establish (Theorem 2.1 (1)) that, in $\mathrm{ZF}$, it is provable from $\mathrm{CAC}+\mathrm{UF}(\omega)$ and that it implies the weak choice principle $\mathrm{CAC}_{\mathrm{fin}}$ (Theorem 3.11). In view of Theorem 3.7 or Theorem 3.8, this implication is not reversible in ZF (see Remark 3.3 .

In contrast to the result " $\mathrm{CAC}+\mathrm{UF}(\omega) \Rightarrow\left(\forall X, \mathrm{TPC}\left(2^{X}\right)\right)$ " (Theorem 2.1(1)), if we weaken $\mathrm{CAC}$ to $\mathrm{CMC}$, then the resulting statement fails to be true in $\mathrm{ZF}^{0}$ (i.e., $\mathrm{ZF}$ minus the Axiom of Regularity), that is, there exists a model of $\mathrm{ZF}^{0}$ in which $\mathrm{CMC}+\mathrm{UF}(\omega)$ is valid, hence by Proposition 2.4 "for every infinite set $X$, every countably infinite subset of $2^{X}$ has an accumulation point" is true in that model, whereas there exists an infinite set $X$ such that $2^{X}$ fails to be countably compact in that model (see the paragraph before Lemma 3.1). In contrast to the previous result, we show (Theorem 3.10 that if we restrict ourselves to $X=\mathbb{R}$, then under CMC, the statements "every countably infinite subset of $2^{\mathbb{R}}$ has an accumulation point", "every infinite subset of $2^{\mathbb{R}}$ has an accumulation point", $\operatorname{TPC}\left(2^{\mathbb{R}}\right)$, and $\operatorname{UF}(\omega)$ are pairwise equivalent.

Before setting out the main results of the paper, let us first provide some preliminary results we shall need.

Proposition 2.1 ([6]). (ZF) For every well-ordered cardinal number , the Tychonoff product $2^{\aleph}$ is compact.

Proposition 2.2 ([3]). Assume CAC. Then a topological space $(X, T)$ is countably compact iff it is sequentially accumulation point compact. 
Proposition 2.3 ([2, Proposition 2.2(3)]). (ZF) Every Tychonoff product of ultrafilter-compact $\mathrm{T}_{2}$ spaces is ultrafilter-compact. In particular, for every infinite set $X$, the Tychonoff product $2^{X}$ is ultrafilter-compact.

Proposition 2.4. In $\mathrm{ZF}, \mathrm{UF}(\omega)$ implies that for every infinite set $X$, every countably infinite subset of $2^{X}$ has an accumulation point. Hence, $\mathrm{UF}(\omega)$ implies that for every infinite set $X$, the Tychonoff product $2^{X}$ has no countably infinite closed relatively discrete subsets.

Proof. Fix an infinite set $X$ and let $A=\left\{a_{n}: n \in \omega\right\}$ be a countably infinite subset of $2^{X}$. By $\operatorname{UF}(\omega)$, let $\mathcal{F}$ be a free ultrafilter on $A$. Put

$$
\mathcal{G}=\left\{Y \subseteq 2^{X}: Y \cap A \in \mathcal{F}\right\} .
$$

It can be readily verified that $\mathcal{G}$ is an ultrafilter on $2^{X}$. Since $2^{X}$ is ultrafiltercompact (see Proposition 2.3), $\mathcal{G}$ converges to a point $g \in 2^{X}$. Since $\mathcal{F}$ is free, it follows that for every open neighborhood $O_{g}$ of $g, O_{g} \cap A$ is an infinite set. Hence, $g$ is an accumulation point of $A$, finishing the proof.

Proposition 2.5. Assume CAC. Then the following statements are pairwise equivalent:

(1) For every infinite set $X, \operatorname{TPC}\left(2^{X}\right)$.

(2) For every infinite set $X$, every infinite subset of $2^{X}$ has an accumulation point.

(3) For every infinite set $X$, every countably infinite subset of $2^{X}$ has an accumulation point.

Proof. (1) $\Rightarrow(2)$. Fix an infinite set $X$ and an infinite set $A \subseteq 2^{X}$. By CAC, $A$ has a countably infinite subset, say $B$. By Proposition 2.2, $B$ has an accumulation point, say $g \in 2^{X}$. Clearly, $g$ is also an accumulation point of $A$, finishing the proof of the implication.

$(2) \Rightarrow(3)$. This is straightforward.

$(3) \Rightarrow(1)$. This follows from Proposition 2.2

THEOREM 2.1.

(1) $\mathrm{CAC}+\mathrm{UF}(\omega)$ implies "for every infinite set $X, \operatorname{TPC}\left(2^{X}\right)$ ".

(2) In every Fraenkel-Mostowski permutation model, CAC implies "for every infinite set $X, \operatorname{TPC}\left(2^{X}\right) "$.

Proof. (1) The statement follows from Propositions 2.2 and 2.4.

(2) This follows from (1) and the fact that $\operatorname{UF}(\omega)$ holds in every permutation model; see [4].

Proposition 2.6 (77, Proposition 3]). (ZF) If $|X|=|Y|$ (i.e., there is a bijection $f: X \rightarrow Y)$, then the Tychonoff products $2^{X}$ and $2^{Y}$ are topologically homeomorphic. 


\section{Main results}

3.1. The Tychonoff product $2^{\mathbb{R}}$. In this part we prove that the choice principle $\mathrm{AC} \mathrm{CO}^{\mathrm{WO}}$ does not imply that $2^{\mathbb{R}}$ is countably compact in ZF. First, we show why the weak choice principle $\mathrm{CAC}(\mathbb{R})$ suffices in order to show that the family of Lebesgue measurable sets is a $\sigma$-algebra. We begin by observing that $\mathrm{CAC}(\mathbb{R})$ implies the $\sigma$-subadditivity of the outer measure $m^{*}$ (for definitions see [12]).

Theorem 3.1. Assume $\mathrm{CAC}(\mathbb{R})$. Let $\left\{A_{n}: n \in \mathbb{N}\right\}$ be a family of sets of reals. Then $m^{*}\left(\bigcup_{n \in \mathbb{N}} A_{n}\right) \leq \sum_{n=1}^{\infty} m^{*}\left(A_{n}\right)$.

Proof. We follow the proof in $[12$ indicating the use of $\mathrm{CAC}(\mathbb{R})$. First note that if $m^{*}\left(A_{n}\right)=\infty$ for some $n \in \mathbb{N}$, then the inequality holds trivially. So assume that $m^{*}\left(A_{n}\right)<\infty$ for all $n \in \mathbb{N}$. Let $\epsilon$ be an arbitrary positive number. Let $\mathcal{C}$ denote the collection of all open intervals. For every $I \in \mathcal{C}$, $l(I)$ denotes the length of the interval $I$. By the definition of the outer measure (see [12]) it follows that for each $n \in \mathbb{N}$, the set

$F_{n}=\left\{f \in \mathcal{C}^{\mathbb{N}}: A_{n} \subseteq \bigcup\{f(m): m \in \mathbb{N}\}\right.$ and $\left.\sum_{m} l(f(m))<m^{*}\left(A_{n}\right)+\frac{\epsilon}{2^{n}}\right\}$

is non-empty. It is well-known that $|\mathcal{C}|=2^{\aleph_{0}}$ and $\left|\mathbb{R}^{\mathbb{N}}\right|=2^{\aleph_{0}}$, hence we may view each $F_{n}$ as a non-empty set of reals. By $\mathrm{CAC}(\mathbb{R})$, let $\left\{\left(n, f_{n}\right): n \in \mathbb{N}\right\}$ be a choice function of the family $\left\{F_{n}: n \in \mathbb{N}\right\}$. Then $\left\{f_{n}(i): n, i \in \mathbb{N}\right\}$ is a countable collection of open intervals whose union covers $\bigcup A_{n}$. Thus,

$$
\begin{aligned}
m^{*}\left(\bigcup A_{n}\right) & \leq \sum_{n, i} l\left(f_{n}(i)\right)=\sum_{n} \sum_{i} l\left(f_{n}(i)\right)<\sum_{n}\left(m^{*}\left(A_{n}\right)+\frac{\epsilon}{2^{n}}\right) \\
& =\sum_{n} m^{*}\left(A_{n}\right)+\epsilon .
\end{aligned}
$$

Since $\epsilon$ was arbitrary, we have the result.

Using the $\sigma$-subadditivity of the outer measure (and assuming $\mathrm{CAC}(\mathbb{R})$ ) one shows that the collection $\mathcal{M}$ of all (Carathéodory) measurable sets is a $\sigma$-algebra (see [12, Corollary 8, Lemma 9, Theorem 10]). Then one defines the Lebesgue measure $m$ on $\mathcal{M}$ by requiring $\forall A \in \mathcal{M}, m(A)=m^{*}(A)$. We would like to point out that in the absence of $C A C(\mathbb{R})$ one may not be able to define the Lebesgue measure. To see this, recall the Feferman-Levy model (model $\mathcal{M} 9$ in [4]) in which $\mathbb{R}$ is expressed as a countable union of countable sets. Since $\mathrm{CAC}(\mathbb{R})$ implies the countable union of countable sets of reals is countable, we see that $\mathrm{CAC}(\mathbb{R})$ fails in this model. If the Lebesgue measure $m$ could be defined in it, then $+\infty=m(\mathbb{R})=0$ (the latter equality would follow from the fact that the outer measure $m^{*}$ of a singleton is zero, hence by the $\sigma$-subadditivity of $m^{*}, m^{*}(A)=0$ for every countable set $A \subseteq \mathbb{R}$; 
therefore in this model, even $m^{*}$ is not $\sigma$-subadditive and consequently $m$ cannot be $\sigma$-additive). The above discussion serves to justify what follows and concerns the $\sigma$-algebra of measurable sets and the $\sigma$-additivity of the Lebesgue measure.

Definition 3.1. Let $b$ be a positive integer greater than 1 . A sequence of the form

$$
\frac{d_{1}}{b}, \frac{d_{2}}{b^{2}}, \ldots, \frac{d_{n}}{b^{n}}, \ldots
$$

in which each $d_{i}$ is an integer with $0 \leq d_{i} \leq b-1$ is called a development with base $b$. When $b=2$ we have a binary development and $d_{i}$ is 0 or 1 for all $i$.

If, in a development with base $b$, only a finite number of the $d_{i}$ are different from 0 , then the development is called terminating; otherwise it is called non-terminating.

If there exists an integer $i_{0}$ such that $d_{i}=b-1$ for all $i>i_{0}$, then we say the development is improper; otherwise it is called proper.

For the proof of the next couple of theorems we refer the reader to [11.

Theorem 3.2. (ZF) Consider an arbitrary binary development $\left(d_{i} / 2^{i}\right)_{i \in \mathbb{N}}$ and define $s_{n}=\sum_{i=1}^{n} d_{i} / 2^{i}, n=1,2, \ldots$ Then:

(1) $\lim _{n \rightarrow \infty} s_{n}=a$ where $a \in[0,1]$.

(2) $a=1$ if and only if $d_{i}=1$ for every $i=1,2, \ldots$.

(3) If the development is improper, then there are positive integers $n$ and $p$ such that $a=p / 2^{n}$ with $p \leq 2^{n}$.

TheOREM 3.3. (ZF) Let $a \in[0,1)$. Then there is a unique proper binary development $\left(d_{i} / 2^{i}\right)_{i \in \mathbb{N}}$ such that $\lim _{n \rightarrow \infty} s_{n}=a$, where $s_{n}=\sum_{i=1}^{n} d_{i} / 2^{i}$, $d_{i} \in\{0,1\}$ for all $i \in \mathbb{N}$.

Definition 3.2. A subset $X$ of $2^{\mathbb{N}}$ is called a tail set if whenever $x \in X$ and $y \in 2^{\mathbb{N}}$ differs from $x$ in a finite number of coordinates only, then $y \in X$.

The mapping $g: 2^{\mathbb{N}} \rightarrow[0,1]$ defined by $g\left(\left(x_{i}\right)_{i \in \mathbb{N}}\right)=\sum_{i=1}^{\infty} x_{i} / 2^{i}$ is onto (not one-to-one) and defines a measure $\mu(E)=m(g(E))$ on the class $\left\{E \subseteq 2^{\mathbb{N}}: g(E)\right.$ is Lebesgue measurable $\}$, where $m$ is the Lebesgue measure. $\mu$ can be taken as the definition of the product measure in $2^{\mathbb{N}}$; see [10].

According to the zero-one law of Kolmogoroff for the product measure, the following result holds; for its proof the reader is referred to [10, Theorem 21.3 , p. 84].

THEOREM 3.4. If $E$ is a measurable tail set in $2^{\mathbb{N}}$, then either $\mu(E)=0$ or $\mu(E)=1$. 
THEOREM 3.5. In $\mathrm{ZF}$, the following statements are pairwise equivalent:

(i) $\operatorname{UF}(\omega)$.

(ii) For every infinite set $X$, every countably infinite subset of $2^{X}$ has an accumulation point.

(iii) Every countably infinite subset of $2^{\mathbb{R}}$ has an accumulation point.

Proof. (i) $\Rightarrow$ (ii). This has been established in Proposition 2.4.

(ii) $\Rightarrow$ (iii). This is straightforward.

(iii) $\Rightarrow$ (i). First note that since $|\mathcal{P}(\mathbb{N})|=|\mathbb{R}|$ in $\mathrm{ZF}$, in view of Proposition 2.6 we may assume that every countably infinite subset of the Tychonoff product $2^{\mathcal{P}(\mathbb{N})}$ has an accumulation point.

Now, for every $n \in \mathbb{N}$, let $f_{n}$ be the function from $\mathcal{P}(\mathbb{N})$ into 2 defined as follows:

$$
f_{n}(A)=1 \Leftrightarrow n \in A,
$$

for every $A \in \mathcal{P}(\mathbb{N})$. In other words, $f_{n}$ codes the principal ultrafilter on $\mathbb{N}$

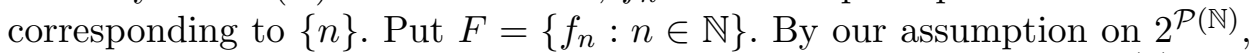
the countably infinite set $F$ has an accumulation point, say $g \in 2^{\mathcal{P}(\mathbb{N})}$. We assert that $g$ codes a free ultrafilter on $\mathbb{N}$. In particular, let $\mathcal{U}=g^{-1}(\{1\})$. We show that $\mathcal{U}$ is a free ultrafilter on $\mathbb{N}$.

1. We show that $\mathbb{N} \in \mathcal{U}$. If not, then $g(\mathbb{N})=0$. Consider the open neighborhood $O_{g}=[\{(\mathbb{N}, 0)\}]$ of $g$ and let $n \in \mathbb{N}$ be such that $f_{n} \in O_{g}$. Then $f_{n}(\mathbb{N})=0$, hence by the definition of $f_{n}$ we have $n \notin \mathbb{N}$, a contradiction. Thus, $\mathbb{N} \in \mathcal{U}$ as asserted. Similarly, one can show that $\emptyset \notin \mathcal{U}$.

2. Let $A, B \in \mathcal{U}$. Then $g(A)=g(B)=1$. We show that $A \cap B \in \mathcal{U}$. Assume the contrary; then $g(A \cap B)=0$ and we consider the open neighborhood $O_{g}=[\{(A, 1),(B, 1),(A \cap B, 0)\}]$ of $g$. Since $g$ is an accumulation point of the set $F$, let $n \in \mathbb{N}$ be such that $f_{n} \in O_{g}$. From this fact and the definition of $f_{n}$ we deduce that $n \in A \cap B$ and $n \notin A \cap B$. This is a contradiction, hence $A \cap B \in \mathcal{U}$ as required.

3. Let $A \in \mathcal{U}$ (hence $g(A)=1$ ) and let $B \in \mathcal{P}(\mathbb{N})$ be such that $A \subseteq B$. We show that $B \in \mathcal{U}$. Assume the contrary; then $g(B)=0$. Consider the open neighborhood $O_{g}=[\{(A, 1),(B, 0)\}]$ of $g$ and let $n \in \mathbb{N}$ be such that $f_{n} \in O_{g}$. By the definition of $f_{n}$ it follows that $n \in A-B$, which contradicts the fact that $A \subseteq B$. Hence, $B \in \mathcal{U}$ as required.

From the above we conclude that $\mathcal{U}$ is a filter on $\mathbb{N}$.

4. For every $A \in \mathcal{P}(\mathbb{N})$, either $A \in \mathcal{U}$ or $A^{c} \in \mathcal{U}$. Assume the contrary and let $A \in \mathcal{P}(\mathbb{N})$ be such that $g(A)=g\left(A^{c}\right)=0$. Consider the open neighborhood $O_{g}=\left[\left\{(A, 0),\left(A^{c}, 0\right)\right\}\right]$ of $g$ and let $n \in \mathbb{N}$ be such that $f_{n} \in O_{g}$. Then $n \notin A \cup A^{c}=\mathbb{N}$, a contradiction. Thus, $\mathcal{U}$ is an ultrafilter on $\mathbb{N}$. 
5. $\cap \mathcal{U}=\emptyset$. If not, then since $\mathcal{U}$ is an ultrafilter on $\mathbb{N}$, we may conclude that $\mathcal{U}$ is the principal ultrafilter on $\mathbb{N}$ corresponding to $\{n\}$ for some $n \in \mathbb{N}$. Since $\{n\} \in \mathcal{U}$ we have $g(\{n\})=1$. Consider the open neighborhood $O_{g}=[\{(\{n\}, 1)\}]$ of $g$. Since $g$ is an accumulation point of the set $F=\left\{f_{n}: n \in \mathbb{N}\right\}$ and $2^{\mathcal{P}(\mathbb{N})}$ is a Hausdorff space, it follows that $O_{g}$ meets $F$ in an infinite set. However, $O_{g} \cap F=\left\{f_{n}\right\}$. This is a contradiction, hence $\mathcal{U}$ is a free ultrafilter on $\mathbb{N}$.

This completes the proof of the implication and of the theorem.

Corollary 3.1. In $\mathrm{ZF}$, the statement "TPC $\left(2^{X}\right)$ for every infinite set $X$ ” implies $\operatorname{UF}(\omega)$. In particular, $\operatorname{TPC}\left(2^{\mathbb{R}}\right)$ implies $\operatorname{UF}(\omega)$.

TheOREM 3.6. Assume $\mathrm{CAC}(\mathbb{R})+$ "every countably infinite subset of $2^{\mathbb{R}}$ has an accumulation point". Then there exists a subset of $[0,1]$ which is not Lebesgue measurable.

Proof. It is well-known (see [5, Problem 10, p. 7]) that $\mathrm{UF}(\omega)$ (which, by Theorem 3.5. is equivalent to "every countably infinite subset of $2^{\mathbb{R}}$ has an accumulation point") implies the existence of a subset $A$ of $2^{\mathbb{N}}$ such that:

1. $\forall x \in 2^{\mathbb{N}}, x \in A$ if and only if $x^{*}=1-x \notin A$, where 1 is the constant function $f(n)=1$ for all $n \in \mathbb{N}$,

2. $A$ is a tail set in $2^{\mathbb{N}}$.

In particular, $A=\left\{\chi_{U}: U \in \mathcal{U}\right\}$, where $\chi_{U}$ is the characteristic function of $U$ and $\mathcal{U}$ is a free ultrafilter on $\mathbb{N}$. Let $\mu$ be the product measure in $2^{\mathbb{N}}$. Now, if $A$ were a $\mu$-measurable set, then $A^{c}=\left\{a^{*}: a \in A\right\}=\{1-a: a \in A\}$ (due to statement 1 ) would also be $\mu$-measurable, and we should have $\mu(A)=$ $\mu\left(A^{c}\right)=1 / 2$. Indeed, let $B=g[A]=\left\{y \in[0,1]: y=\sum_{n=1}^{\infty} x_{n} / 2^{n}\right.$ for some $\left.\left(x_{n}\right)_{n \in \mathbb{N}} \in A\right\}$, where $g$ is the function defined in the paragraph before Theorem 3.4. Then $B^{c}=g\left[A^{c}\right]=\left\{y \in[0,1]: y=\sum_{n=1}^{\infty}\left(1-x_{n}\right) / 2^{n}\right.$ for some $\left.\left(x_{n}\right)_{n \in \mathbb{N}} \in A\right\}=\{1-b: b \in B\}=1-B$. [To see this, let $y \in[0,1]-B$. If $y=1$, then $y=\sum_{n=1}^{\infty} 1 / 2^{n}$ and the sequence $\left(x_{n}\right)_{n \in \mathbb{N}}$, where $x_{n}=1$ for all $n \in \mathbb{N}$, does not belong to $A$. Since $A$ satisfies property 1 above, we find that the sequence $\left(y_{n}\right)_{n \in \mathbb{N}}$, where $y_{n}=0$ for all $n \in \mathbb{N}$, belongs to $A$. Hence, $\sum_{n=1}^{\infty} y_{n} / 2^{n} \in B$ and $y=1-\sum_{n=1}^{\infty} y_{n} / 2^{n} \in 1-B$. If $y \in[0,1)$, let $\left(d_{n} / 2^{n}\right)_{n \in \mathbb{N}}$ be the unique proper binary development of $y$ (see Theorem 3.3. Then $y=\sum_{n=1}^{\infty} d_{n} / 2^{n}$ and $\left(d_{n}\right)_{n \in \mathbb{N}} \notin A$ (recall that $y \notin B$ ). Thus, by item $1,\left(1-d_{n}\right)_{n \in \mathbb{N}} \in A$, and $y=1-\sum_{n=1}^{\infty}\left(1-d_{n}\right) / 2^{n} \in 1-B$. Hence, $B^{c} \subseteq 1-B$. Now, let $y \in 1-B$. Then $y=1-\sum_{n=1}^{\infty} x_{n} / 2^{n}$ for some $\left(x_{n}\right)_{n \in \mathbb{N}} \in A$. By way of contradiction assume that $y \in B$, hence $y=$ $\sum_{n=1}^{\infty} y_{n} / 2^{n}$ for some $\left(y_{n}\right)_{n \in \mathbb{N}} \in A$. Then $1-\sum_{n=1}^{\infty} x_{n} / 2^{n}=\sum_{n=1}^{\infty} y_{n} / 2^{n}$, hence $1=\sum_{n=1}^{\infty}\left(x_{n}+y_{n}\right) / 2^{n}$. By Theorem $3.2(2)$ we have $x_{n}+y_{n}=1$ for all $n \in \mathbb{N}$, hence $x_{n}=1-y_{n}$ for all $n \in \mathbb{N}$ and since $\left(x_{n}\right)_{n \in \mathbb{N}} \in A$, it follows that $\left(1-y_{n}\right)_{n \in \mathbb{N}} \in A$. As $\left(y_{n}\right)_{n \in \mathbb{N}} \in A$ it follows, by property 1 , that 
$\left(1-y_{n}\right)_{n \in \mathbb{N}} \notin A$, a contradiction. Thus, $y \in B^{c}$ and $1-B \subseteq B^{c}$. Thus, $B^{c}=1-B$ as required.]

So, if $B$ is Lebesgue measurable, then so is $B^{c}$ and since for any set $X \subset \mathbb{R}, m^{*}(X)=m^{*}(-X)$, where $m^{*}$ is the outer measure and $-X=$ $\{-x: x \in X\}\left(m^{*}(X)=m^{*}(-X)\right.$ follows easily from the definition of the outer measure $\left.m^{*}\right)$, and the Lebesgue measure is translation invariant, we see that $m(B)=m(-B)=m(-B+1)=m\left(B^{c}\right)$ where $-B+1=\{-b+1$ : $b \in B\}=1-B$. Since $[0,1]=B \cup B^{c}, m([0,1])=1$, and the Lebesgue measure $m$ is $\sigma$-additive $(\mathrm{CAC}(\mathbb{R})$ is needed here; see Theorem 3.1 and [12]), we must have $m(B)=m\left(B^{c}\right)=1 / 2$. Therefore, $\mu(A)=\mu\left(A^{c}\right)=1 / 2$.

Since $A^{c}$ is also a tail set and $\mu(A)=\mu\left(A^{c}\right)>0$, by Kolmogoroff's zero-one law (see Theorem 3.4 we should have $\mu(A)=\mu\left(A^{c}\right)=1$, which is impossible. Consequently, the set $B=g[A] \subset[0,1]$ is not Lebesgue measurable, finishing the proof of the theorem.

Although the result of Theorem 3.8 below is stronger than the result of the next Theorem 3.7, we incorporate both of them since we wish to indicate that $\operatorname{TPC}\left(2^{\mathbb{R}}\right)$ fails in two important forcing models, namely in Feferman's model [1] and in Solovay's model [14].

TheOrem 3.7. If $\mathrm{ZF}$ is consistent, then so is $\mathrm{ZF}+\mathrm{CAC}+$ "the Tychonoff product $2^{\mathbb{R}}$ contains a countably infinite, closed and relatively discrete subset". Consequently, if $\mathrm{ZF}$ is consistent, then so is $\mathrm{ZF}+\mathrm{CAC}+$ "the Tychonoff product $2^{\mathbb{R}}$ is not countably compact".

Proof. In Solovay's forcing model (model $\mathcal{M} 5(\aleph)$ in [4]) the principle of dependent choices $\mathrm{DC}$ is true (and $\mathrm{AC}^{\mathrm{WO}}$ is false), hence the weaker axioms $\mathrm{CAC}$ and $\mathrm{CAC}(\mathbb{R})$ also hold in this model. On the other hand, Solovay's model satisfies "every set of reals is Lebesgue measurable", hence by Theorem 3.6 it follows that in $\mathcal{M} 5(\aleph)$ there exists a countably infinite subset of $2^{\mathbb{R}}$ with no accumulation points (see the proof of Theorem 3.6). Consequently, the statement "the Tychonoff product $2^{\mathbb{R}}$ contains a countably infinite, closed and relatively discrete subset" is true in $\mathcal{M} 5(\aleph)$. Since, in $\mathrm{ZF}$, " $2^{\mathbb{R}}$ is countably compact" implies "every countably infinite subset of $2^{\mathbb{R}}$ has an accumulation point", we deduce that $2^{\mathbb{R}}$ fails to be countably compact in Solovay's model. This completes the proof.

TheOREM 3.8. If $\mathrm{ZF}$ is consistent, then so is $\mathrm{ZF}+\mathrm{AC}^{\mathrm{WO}}+$ "the Tychonoff product $2^{\mathbb{R}}$ is not countably compact".

Proof. Truss [15] shows that in Feferman's forcing model (model $\mathcal{M} 2$ in [4]; see also [1]) the choice principle $\mathrm{AC}^{\mathrm{WO}}$ holds true and that the family of pairs $\mathcal{A}=\{\{[X],[\mathbb{N}-X]\}: X \subseteq \mathbb{N}\}$, where for every $X \subseteq \mathbb{N},[X]$ is the equivalence class of $X$ under the equivalence relation $X \sim Y$ if and only if $|X \triangle Y|<\aleph_{0}$ where $\triangle$ is the symmetric difference operation, does not have 
a choice function in this model. It is easy to see that $\operatorname{UF}(\omega)$ implies that $\mathcal{A}$ has a choice function, thus $\mathrm{UF}(\omega)$ does not hold in Feferman's model. By Corollary 3.1 we also infer that $2^{\mathbb{R}}$ fails to be countably compact in this model. This completes the proof.

From Theorems 3.5, 3.7 (or 3.8) we see that the statement "every countably infinite subset of $2^{\mathbb{R}}$ has an accumulation point" is not provable in ZF. Hence, the stronger statement "every infinite subset of $2^{\mathbb{R}}$ has an accumulation point" is not a theorem of ZF either. In the following theorem we relate the latter proposition to a weak choice form.

THEOREM 3.9. In $\mathrm{ZF}$, if every infinite subset of $2^{\mathbb{R}}$ has an accumulation point, then every countable family of two-element subsets of $\mathcal{P}(\mathbb{R})$ has a choice function. The latter choice principle is not provable in $\mathrm{ZF}$.

Proof. Let $\mathcal{A}=\left\{A_{i}: i \in \mathbb{N}\right\}$ be a family of two-element subsets of $\mathcal{P}(\mathbb{R})$. Without loss of generality we may assume that the collection $\bigcup \mathcal{A}$ is pairwise disjoint. [To see this, first consider the family $\mathcal{B}=\left\{B_{i}: i \in \mathbb{N}\right\}$, where for $i \in \mathbb{N}, B_{i}=\left\{X \times\{i\}: X \in A_{i}\right\}$. Since in ZF it is true that $|\mathbb{R} \times \mathbb{N}|=|\mathbb{R}|$, we may view $\mathcal{B}$ as a (pairwise disjoint) family of subsets of $\mathcal{P}(\mathbb{R})$. Second, for every $i \in \mathbb{N}$, if $B_{i}=\left\{X_{i}, Y_{i}\right\}$, then we may assume that $X_{i}-Y_{i} \neq \emptyset$ and $Y_{i}-X_{i} \neq \emptyset$. Otherwise, we may choose from $B_{i}$ the element $\bigcap B_{i}$. Define $\mathcal{C}=\left\{C_{i}: i \in \mathbb{N}\right\}$, where $C_{i}=\left\{X-Y: X, Y \in B_{i}, X \neq Y\right\}$. Then $\bigcup \mathcal{C}$ is pairwise disjoint and we may work in the proof below with $\mathcal{C}$ instead of $\mathcal{A}$ ]. For each $i \in \mathbb{N}$, let

$$
\begin{aligned}
B_{i}=\left\{f \in 2^{\mathbb{R}}:\left(\forall j \leq i, \exists P \in A_{j},(f[P]=\{1\} \wedge\right.\right. & \left.\left.f\left[\left(\bigcup A_{j}\right) \backslash P\right]=\{0\}\right)\right) \\
& \left.\wedge\left(\forall j>i, f\left[\bigcup A_{j}\right]=\{0\}\right)\right\} .
\end{aligned}
$$

Notice that $B_{i}$ is a finite set for all $i \in \mathbb{N}$. Put $B=\bigcup\left\{B_{i}: i \in \mathbb{N}\right\}$. By our hypothesis, the infinite set $B$ has an accumulation point, say $g$. Then for each $i \in \mathbb{N}, g$ separates the two elements of $A_{i}$, that is, there exists $P \in A_{i}$ such that $g[P]=\{1\}$ and $g\left[\left(\bigcup A_{i}\right) \backslash P\right]=\{0\}$. Indeed, assume the contrary and let $i \in \mathbb{N}$ be such that $g$ does not separate the elements of $A_{i}=\left\{F_{i}, G_{i}\right\}$. We consider the following two cases:

(1) $\exists x \in F_{i}, \exists y \in G_{i}$ such that $g(x)=g(y)=a$ for some $a \in 2$. Consider the basic open neighborhood $O=[\{(x, a),(y, a)\}]$ of $g$. If $a=1$ or $i=1$, then $O \cap B=\emptyset$, which is a contradiction since $g$ being an accumulation point of $B$ in the Hausdorff space $2^{\mathbb{R}}$ has the property that each one of its neighborhoods meets $B$ in an infinite set. Hence, we may assume that $a=0$ and $i>1$. But then $O \cap B=\bigcup\left\{B_{j}: j<i\right\}$, which is a finite set, a contradiction again.

(2) $\exists x, y \in F_{i}$ such that $g(x)=1$ and $g(y)=0$. Then $Q=[\{(x, 1),(y, 0)\}]$ is a neighborhood of $g$ which avoids $B$, a contradiction. 
Thus, $g$ separates the elements of $A_{i}$ for all $i \in \mathbb{N}$ as required. Clearly, $g^{-1}(\{1\})$ is a choice set of $\mathcal{A}$, finishing the proof of the first assertion of the theorem.

For the second assertion, it is known (see [4]) that in Cohen's second model (model $\mathcal{M} 7$ in 4]) there is a countable family of 2 -element subsets of $\mathcal{P}(\mathbb{R})$ which has no infinite subfamily with a choice function. Hence, the statement "every countable family of two-element subsets of $\mathcal{P}(\mathbb{R})$ has a choice function" fails in this model. This completes the proof of the theorem.

In view of Proposition 2.5 we find that $\mathrm{CAC}+$ "for every infinite set $X$, every countably infinite subset of $2^{X}$ has an accumulation point" implies "for every infinite set $X, \operatorname{TPC}\left(2^{X}\right)$ ". However, if we weaken CAC to CMC, then the implication ceases to be true in the setting of $\mathrm{ZF}^{0}$. Indeed, in the second Fraenkel permutation model (model $\mathcal{N} 2$ in [4]), CMC $+\mathrm{UF}(\omega)$ holds (see [4), hence by Proposition 2.4, the statement "for every infinite set $X$, every countably infinite subset of $2^{X}$ has an accumulation point" also holds in $\mathcal{N} 2$. However, "for every infinite set $X, \operatorname{TPC}\left(2^{X}\right)$ " implies $\mathrm{CAC}_{\text {fin }}$ (see Theorem 3.11 below) and the latter fails in $\mathcal{N} 2$; see [4].

On the other hand, if we consider the particular case where $X=\mathbb{R}$, then under CMC, the statements "every countably infinite subset of $2^{\mathbb{R}}$ has an accumulation point", "every infinite subset of $2^{\mathbb{R}}$ has an accumulation point", and $\operatorname{TPC}\left(2^{\mathbb{R}}\right)$ are pairwise equivalent. We establish this in Theorem 3.10. First we need the following lemma.

LEMMA 3.1.

(i) In $\mathrm{ZF}, \mathrm{CMC} \Rightarrow \mathrm{CAC}(\mathbb{R}) \Rightarrow \mathrm{CAC}_{\text {fin }}(\mathcal{P}(\mathbb{R}))$. Hence, in $\mathrm{ZF}, \mathrm{CMC}$ implies that every countable family of non-empty subsets of $\mathcal{P}(\mathbb{R})$ has a choice function.

(ii) In ZF, CMC implies that every infinite subset of $2^{\mathbb{R}}$ has a countably infinite subset. The latter statement is not provable in $\mathrm{ZF}$.

(iii) In $\mathrm{ZF}, \mathrm{CAC}_{\mathrm{fin}}(\mathcal{P}(\mathbb{R}))$ if and only if $\mathrm{CUC}_{\text {fin }}(\mathcal{P}(\mathbb{R}))$.

Proof. (i) Using the standard linear order of $\mathbb{R}$, it is straightforward to verify that $\mathrm{CMC}$ implies $\mathrm{CAC}(\mathbb{R})$.

We prove now that $\mathrm{CAC}(\mathbb{R})$ implies $\operatorname{CAC}_{\text {fin }}(\mathcal{P}(\mathbb{R}))$. Let $\mathcal{A}=\left\{A_{i}: i \in \omega\right\}$ be a family of non-empty finite subsets of $\mathcal{P}(\mathbb{R})$. Similarly to the proof of Theorem 3.9 we may assume without loss of generality that the family $\left\{\bigcup A_{i}: i \in \omega\right\}$ is pairwise disjoint. Furthermore, we may assume that

$$
(\forall i \in \omega)\left(\forall A, B \in A_{i}\right)(A \neq B \Rightarrow A-B \neq \emptyset \wedge B-A \neq \emptyset) .
$$

[Otherwise, for each $i \in \omega$, let $B_{i}=\left\{X \in A_{i}: X\right.$ is $\subset$-maximal $\}$. Then, the family $\mathcal{B}=\left\{B_{i}: i \in \omega\right\}$ is such that for all $i \in \omega$ and for all $A, B \in B_{i}$, if $A \neq B$, then $A-B \neq \emptyset$ and $B-A \neq \emptyset$.] 
Claim. For each $i \in \omega$, there exists a finite subset $W_{i}$ of $\mathbb{R}$ such that for all $A, B \in A_{i}$ with $A \neq B$, it is the case that $A \cap W_{i} \neq B \cap W_{i}$.

Proof of Claim. Fix an $i \in \omega$. For each $(A, B) \in A_{i} \times A_{i}$ such that $A \neq B$, choose an element $x_{(A, B)} \in A \backslash B$. Put

$$
W_{i}=\left\{x_{(A, B)}:(A, B) \in A_{i} \times A_{i}, A \neq B\right\} .
$$

In view of (1) we have $W_{i} \neq \emptyset$ and it is evident that $A \cap W_{i} \neq B \cap W_{i}$ for all $A, B \in A_{i}$ with $A \neq B$.

For each $i \in \omega$, put

$$
B_{i}=\left\{W \in[\mathbb{R}]^{<\omega}:\left(\forall A, B \in A_{i}\right)(A \neq B \Rightarrow A \cap W \neq B \cap W)\right\},
$$

where $[\mathbb{R}]^{<\omega}$ is the set of all finite subsets of $\mathbb{R}$. By the claim we know that $B_{i} \neq \emptyset$ for all $i \in \omega$. Since in ZF, $\left|[\mathbb{R}]^{<\omega}\right|=|\mathbb{R}|$, we may view each $B_{i}$ as a subset of $\mathbb{R}$. Hence, by $\operatorname{CAC}(\mathbb{R})$, let $f$ be a choice function of the family $\mathcal{B}=\left\{B_{i}: i \in \omega\right\}$. Then $F=\bigcup\left\{f\left(B_{i}\right): i \in \omega\right\}$ is countable, being a countable union of finite subsets of $\mathbb{R}$. Put

$$
\mathcal{U}=\left\{A \cap f\left(B_{i}\right): A \in A_{i}, i \in \omega\right\} .
$$

Then $\mathcal{U}$ is countable being a subset of $[F]^{<\omega}$ which is countable since $F$ is countable (recall that in $\mathrm{ZF},|[\omega]<\omega|=\aleph_{0}$ ). Consider now the function $h: \bigcup \mathcal{A} \rightarrow \mathcal{U}$ defined as follows: Let $A \in \bigcup \mathcal{A}$. By our assumption that $\left\{\bigcup A_{i}: i \in \omega\right\}$ is pairwise disjoint, there exists a unique $i \in \omega$ such that $A \in A_{i}$. Put $h(A)=A \cap f\left(B_{i}\right)$. Since for each $i \in \omega, f\left(B_{i}\right)$ satisfies the claim and $\left\{\bigcup A_{i}: i \in \omega\right\}$ is pairwise disjoint, it follows that $h$ is one-to-one. Thus, $\cup \mathcal{A}$ is countable and $\mathcal{A}$ has a choice function as required. This completes the proof of the implication.

The last assertion of (i) is, in view of the above, straightforward. The proof of (i) is complete.

(ii) Assume CMC and let $A$ be an infinite subset of $2^{\mathbb{R}}$. Now, in ZF, $\left|\left(2^{\mathbb{R}}\right)^{\omega}\right|=\left|2^{\mathbb{R} \times \omega}\right|=\left|2^{\mathbb{R}}\right|$, and since for every $n \in \omega$,

$$
\left|\left(2^{\mathbb{R}}\right)^{n}\right|=\left|\left(2^{\mathbb{R}}\right)^{n} \times\{\mathbf{0}\} \times\{\mathbf{0}\} \times \cdots\right| \leq\left|\left(2^{\mathbb{R}}\right)^{\omega}\right|,
$$

where $\mathbf{0}$ is the constant function $f(x)=0$ for all $x \in \mathbb{R}$, we may effectively define (i.e., without choice) an injection from $\left(2^{\mathbb{R}}\right)^{n}$ into $2^{\mathbb{R}}$ for every $n \in \omega$. Thus, for all $n \in \omega$, we may view the elements of $\left(2^{\mathbb{R}}\right)^{n}$ as elements of $2^{\mathbb{R}}$. For each $n \in \omega$, put $I_{n}=\left\{f \in A^{n}: f\right.$ is an injection $\}$. As $A$ is infinite, it follows that $I_{n} \neq \emptyset$ for all $n \in \omega$. By CMC and (i) it follows that the disjoint family $\left\{I_{n}: n \in \omega\right\}$ has a choice set, say $\left\{f_{n}: n \in \omega\right\}$. By induction and using the $f_{n}$ 's we may construct a countably infinite subset of $A$.

For the second assertion of (ii) we recall that in the second Cohen model (model $\mathcal{M} 7$ in [4]) there is a countable family of 2 -element subsets of $\mathcal{P}(\mathbb{R})$ 
which has no infinite subfamily with a choice function. Therefore, the statement "every infinite set $A \subset 2^{\mathbb{R}}$ has a countably infinite subset" fails in this model. This completes the proof of (ii).

(iii) It suffices to show that $\mathrm{CAC}_{\text {fin }}(\mathcal{P}(\mathbb{R})) \Rightarrow \operatorname{CUC}_{\text {fin }}(\mathcal{P}(\mathbb{R}))$ as the reverse implication is evident. Fix a family $\mathcal{A}=\left\{A_{i}: i \in \omega\right\}$ of non-empty finite subsets of $\mathcal{P}(\mathbb{R})$. For every $i \in \omega$, define

$$
B_{i}=\left\{f \in(\mathcal{P}(\mathbb{R}))^{\omega}:\left(f\left[\left|A_{i}\right|\right]=A_{i}\right) \wedge\left(f\left[\omega \backslash\left|A_{i}\right|\right]=\{\emptyset\}\right)\right\} .
$$

Clearly, $B_{i}$ is a non-empty finite subset of $(\mathcal{P}(\mathbb{R}))^{\omega}$. Furthermore, since in $\mathrm{ZF},\left|(\mathcal{P}(\mathbb{R}))^{\omega}\right|=\left|\left(2^{\mathbb{R}}\right)^{\omega}\right|=\left|2^{\mathbb{R}}\right|=|\mathcal{P}(\mathbb{R})|$, we may view $B_{i}, i \in \omega$, as a non-empty finite subset of $\mathcal{P}(\mathbb{R})$. Therefore, applying $\operatorname{CAC}_{\text {fin }}(\mathcal{P}(\mathbb{R}))$ to the family $\mathcal{B}=\left\{B_{i}: i \in \omega\right\}$ we obtain a choice function $f$ of $\mathcal{B}$. On the basis of the functions $\left.f(i)\right|_{\left|A_{i}\right|}$ we may easily construct an enumeration of $\bigcup \mathcal{A}$. Therefore, $\bigcup \mathcal{A}$ is countable as required. This completes the proof of (iii) and of the theorem.

REMARK 3.1. In [4] it is stated as unknown whether CMC implies the weak choice principle "every family $\mathcal{A}=\left\{A_{i}: i \in \omega\right\}$ such that $0 \neq\left|A_{i}\right| \leq$ $2^{\left(2^{\aleph_{0}}\right)}$ for all $i \in \omega$, has a choice function". In view of Lemma 3.1 (i), we partially fill this gap.

Theorem 3.10. Assume CMC. Then the following statements are pairwise equivalent:

(i) $\operatorname{TPC}\left(2^{\mathbb{R}}\right)$.

(ii) Every countably infinite subset of $2^{\mathbb{R}}$ has an accumulation point.

(iii) Every infinite subset of $2^{\mathbb{R}}$ has an accumulation point.

(iv) $\mathrm{UF}(\omega)$.

Proof. (i) $\Rightarrow$ (ii). This follows from the fact that in ZF, every countably compact is sequentially accumulation point compact; see Proposition 2.2 .

(ii) $\Rightarrow$ (iii). Fix an infinite set $A \subset 2^{\mathbb{R}}$. By Lemma 3.1 (ii), $A$ has a countably infinite subset, say $B$. Clearly, any accumulation point of $B$ is also an accumulation point of $A$.

(iii) $\Rightarrow$ (i). Let $\mathcal{G}=\left\{G_{n}: n \in \omega\right\}$ be a nested family of non-empty closed subsets of $2^{\mathbb{R}}$. By way of contradiction, assume that $\bigcap \mathcal{G}=\emptyset$. By CMC and Lemma 3.1(i), the disjoint family $\left\{G_{n} \backslash G_{n+1}: n \in \omega\right\}$ has a choice set, say $G=\left\{g_{n}: n \in \omega\right\}$. Clearly, $G$ is a countably infinite, closed and relatively discrete subset of $2^{\mathbb{R}}$. This contradicts our hypothesis that every infinite subset of $2^{\mathbb{R}}$ has an accumulation point. Therefore, $\bigcap \mathcal{G} \neq \emptyset$ and $2^{\mathbb{R}}$ is countably compact as required. This completes the proof of the implication.

(ii) $\Leftrightarrow($ iv) is established in Theorem 3.5 . 
REMARK 3.2. In view of Theorems 2.1(1) and 3.5 we infer that under $\mathrm{CAC}$, the statements "for every infinite set $X, \operatorname{TPC}\left(2^{X}\right)$ " and $\operatorname{UF}(\omega)$ are equivalent.

3.2. Arbitrary Tychonoff powers of 2. In this part of the paper we study the deductive strength of the statement "for every infinite set $X, 2^{X}$ is countably compact" in terms of weak choice principles.

THEOREM 3.11. Each of the following statements implies the one beneath it:

(i) For every infinite set $X, 2^{X}$ is countably compact.

(ii) $\mathrm{CAC}_{\text {fin }}$.

(iii) For every infinite set $X$, every countable basic open cover of $2^{X}$ has a finite subcover.

(iv) For every $n \in \mathbb{N}, \mathrm{CAC}_{\leq n}$ (= Every countable family of $\leq n$-sized non-empty sets has a choice function).

Proof. (i) $\Rightarrow$ (ii). Let $\mathcal{A}=\left\{A_{i}: i \in \omega\right\}$ be a disjoint family of non-empty finite sets. By our hypothesis, the Tychonoff product $2^{X}$, where $X=\bigcup \mathcal{A}$, is countably compact. For each $i \in \omega$, let

$$
B_{i}=\left\{f \in 2^{X}:(\forall j \leq i)\left|f^{-1}(\{1\}) \cap A_{j}\right|=1\right\} .
$$

For every $i \in \omega, B_{i}$ is a non-empty closed subset of $2^{X}$. Indeed, let $i \in \omega$ and $f \in 2^{X} \backslash B_{i}$. Then for some $j \leq i,\left|f^{-1}(\{1\}) \cap A_{j}\right| \neq 1$. There are two cases:

(a) $A_{j} \subseteq f^{-1}(\{0\})$. Then $O_{f}=\left[\left\{(x, 0): x \in A_{j}\right\}\right]$ is a neighborhood of $f$ which does not meet $B_{i}$.

(b) $\left|f^{-1}(\{1\}) \cap A_{j}\right| \geq 2$. Let $x, y \in A_{j}$ be such that $f(x)=f(y)=1$. Consider the neighborhood $O_{f}=[\{(x, 1),(y, 1)\}]$ of $f$. Clearly, $O_{f} \cap B_{i}=\emptyset$.

Therefore, $B_{i}$ is closed in $2^{X}$ and clearly it is non-empty. Furthermore, $\mathcal{B}=\left\{B_{i}: i \in \omega\right\}$ is a descending family, thus, by the countable compactness of $2^{X}$, there exists an element $g \in \cap \mathcal{B}$. Then $C_{i}=g^{-1}(\{1\}) \cap A_{i}$ is a singleton for every $i \in \omega$ and $C=\bigcup\left\{C_{i}: i \in \omega\right\}$ is a choice set of $\mathcal{A}$. This completes the proof of the implication.

(ii) $\Rightarrow$ (iii). Let $X$ be an infinite set and let $\mathcal{U}=\left\{\left[p_{i}\right]: i \in \omega\right\}$ be a basic open cover of $2^{X}$. By $\mathrm{CAC}_{\text {fin }}$, the set $D=\bigcup\left\{\operatorname{Dom}\left(p_{i}\right): i \in \omega\right\}$ is countable, hence $2^{D}$ is compact; see Proposition 2.1. Let $\mathcal{V}=\left\{V_{i}: i \in \omega\right\}$ with $V_{i}=\left\{f \in 2^{D}: p_{i} \subseteq f\right\}$. Then $\mathcal{V}$ is an open cover of $2^{D}$, hence it has a finite subcover, say $\left\{V_{i_{1}}, \ldots, V_{i_{n}}\right\}$. Consequently, $\mathcal{W}=\left\{\left[p_{i_{1}}\right], \ldots,\left[p_{i_{n}}\right]\right\}$ is a finite subcover of $\mathcal{U}$, finishing the proof of the implication.

(iii) $\Rightarrow$ (iv). We prove only that (iii) $\Rightarrow \mathrm{CAC}_{2}$ (i.e., CAC restricted to families of 2-element sets) and by an easy induction this can be generalized. 
Let $\mathcal{A}=\left\{A_{i}: i \in \omega\right\}$ be a disjoint family of pairs. Assume that $\mathcal{A}$ has no choice function. Let

$$
\mathcal{U}=\left\{\left[p_{i, a}\right]: i \in \omega, a \in 2\right\},
$$

where $p_{i, a}$ is the partial function on $A_{i}$ which is constant with value $a$. Since $\mathcal{A}$ has no choice function, $\mathcal{U}$ is a cover of $2 \cup \mathcal{A}$. However, $\mathcal{U}$ does not have any finite subcover, contradicting the statement of (iii) regarding the infinite set $X=\bigcup \mathcal{A}$. The proof of the implication, as well as of the theorem, is complete.

REMARK 3.3. In view of Theorem 3.7 none of the statements given by (ii), (iii), and (iv) in Theorem 3.11 implies "for every infinite set $X, 2^{X}$ is countably compact" in ZF set theory (each of (ii), (iii), and (iv) holds in Solovay's model $\left(\mathcal{M} 5(\aleph)\right.$ in [4]) whereas $2^{\mathbb{R}}$ fails to be countably compact in this model). This is in striking contrast with the fact that, in $\mathrm{ZF}$, the following statements are pairwise equivalent (see [8]):

1. For every infinite set $X, 2^{X}$ is compact (if and only if BPI, i.e., every Boolean algebra has a prime ideal and Form 14 in [4]; see [9]).

2. For every infinite set $X, 2^{X}$ is Lindelöf (i.e., every open cover of $2^{X}$ has a countable subcover).

3. For every infinite set $X$, every cover of $2^{X}$ consisting of basic open sets has a countable subcover.

Acknowledgement. We would like to thank the anonymous referee for some useful comments which improved our paper and in particular for suggesting Theorem 3.5 which simplified the original proof of Theorem 3.6

\section{References}

[1] S. Feferman, Some applications of the notions of forcing and generic sets, Fund. Math. 56 (1965), 325-345.

[2] H. Herrlich, Compactness and the Axiom of Choice, Appl. Categ. Structures 4 (1996), 1-14.

[3] P. Howard, K. Keremedis, J. E. Rubin, and A. Stanley, Compactness in countable Tychonoff products and choice, Math. Logic Quart. 46 (2000), 3-16.

[4] P. Howard and J. E. Rubin, Consequences of the Axiom of Choice, Math. Surveys Monogr. 59, Amer. Math. Soc., 1998 (see also http://consequences.emich.edu).

[5] T. J. Jech, The Axiom of Choice, North-Holland, Amsterdam, 1973. Reprint: Dover Publ., Mineola, NY, 2008.

[6] K. Keremedis, The compactness of $2^{\mathbb{R}}$ and some weak forms of the axiom of choice, Math. Logic Quart. 46 (2000), 569-571.

[7] K. Keremedis, E. Felouzis, and E. Tachtsis, On the compactness and countable compactness of $2^{\mathbb{R}}$ in ZF, Bull. Polish Acad. Sci. Math. 55 (2007), 293-302.

[8] K. Keremedis and E. Tachtsis, Extensions of compactness of Tychonoff powers of 2 in ZF, Topology Proc. 37 (2011), 15-31 (e-published on April 29, 2010). 
[9] J. Mycielski, Two remarks on Tychonoff's product theorem, Bull. Acad. Polon. Sci. Sér. Sci. Math. Astronom. Phys. 12 (1964), 439-441.

[10] J. C. Oxtoby, Measure and Category, Springer, New York, 1980.

[11] M. H. Protter and C. B. Morrey, A First Course in Real Analysis, 2nd ed., Springer, New York, 1991.

[12] H. L. Royden, Real Analysis, 3rd ed., Macmillan, New York, 1988.

[13] W. Sierpiński, Fonctions additives non complètement additives et fonctions non mesurables, Fund. Math. 30 (1938), 96-99.

[14] R. M. Solovay, A model of set theory in which every set of reals is Lebesgue measurable, Ann. of Math. 92 (1970), 1-56.

[15] J. K. Truss, The axiom of choice for linearly ordered families, Fund. Math. 99 (1978), 133-139.

Eleftherios Tachtsis

Department of Statistics and Actuarial-Financial Mathematics

University of the Aegean

Karlovassi, Samos, 83200, Greece

E-mail: ltah@aegean.gr

Received June 24, 2010;

received in final form August 11, 2010 